INPLASY

PROTOCOL

To cite: Zhang et al. Impact of adaptive design on reducing the duration of clinical trials in rare cancers: a meta-analysis. Inplasy protocol 202220081. doi:

10.37766/inplasy2022.2.0081

Received: 19 February 2022

Published: 20 February 2022

Corresponding author:

Cheng Zhang

zhangc_tcm@foxmail.com

Author Affiliation:

School of Pharmaceutical Sciences, Tsinghua University Institute of Pharmaceutical

Regulatory Science.

Support: None.

Review Stage at time of this submission: Preliminary searches.

Conflicts of interest:

None declared.

\section{Impact of adaptive design on reducing the duration of clinical trials in rare cancers: a meta-analysis}

Review question / Objective: Whether the application of adaptive design in clinical trials of rare cancers can shorten the duration of clinical trials?

Condition being studied: Currently, the development of innovative drug products (InMPs) for rare cancers faces many challenges, including the difficulty of enrolling sufficient numbers of patients from small and heterogeneous patient populations for clinical trials, and the significant risks of high financial investment, long development times and potential failure from a pharmaceutical company's perspective for rare cancer drugs due to limited knowledge of the natural history of the disease. Therefore, alternative approaches to clinical trial design are needed to conduct cost-effective, wellcontrolled analyses that can assess treatment effects in small, heterogeneous populations within shorter time frames. Adaptive trials, on the other hand, may be an effective solution to this problem. Adaptive clinical trials are designed to accelerate the clinical trial process by making predefined adjustments to key parameters through data accumulated at predefined time points during the trial without compromising the integrity and validity of the results.This study aims to examine the value of adaptive design in reducing the duration of clinical trials in rare cancers and encourage their wider implementation.

INPLASY registration number: This protocol was registered with the International Platform of Registered Systematic Review and Meta-Analysis Protocols (INPLASY) on 20 February 2022 and was last updated on 20 February 2022 (registration number INPLASY202220081).

\section{INTRODUCTION}

Review question / Objective: Whether the application of adaptive design in clinical trials of rare cancers can shorten the duration of clinical trials? 
Condition being studied: Currently, the development of innovative drug products (InMPs) for rare cancers faces many challenges, including the difficulty of enrolling sufficient numbers of patients from small and heterogeneous patient populations for clinical trials, and the significant risks of high financial investment, long development times and potential failure from a pharmaceutical company's perspective for rare cancer drugs due to limited knowledge of the natural history of the disease. Therefore, alternative approaches to clinical trial design are needed to conduct costeffective, well-controlled analyses that can assess treatment effects in small, heterogeneous populations within shorter time frames. Adaptive trials, on the other hand, may be an effective solution to this problem. Adaptive clinical trials are designed to accelerate the clinical trial process by making predefined adjustments to key parameters through data accumulated at predefined time points during the trial without compromising the integrity and validity of the results.This study aims to examine the value of adaptive design in reducing the duration of clinical trials in rare cancers and encourage their wider implementation.

\section{METHODS}

Search strategy: We comprehensively searched five Chinese databases: China National Knowledge Infrastructure (CNKI), China Biology Medicine (CBM), WanFang Data, VIP, and Chinese Clinical Trial Registry (ChiCTR), and five English databases: PubMed, The Cochrane Library, Embase, Web of Science, and ClinicalTrials.gov. The retrieval time was from the establishment of the database to 12 February, 2022, using the keywords "rare cancer"or "rare tumor"or "rare oncology". The CBM, PubMed, The Cochrane Library, and Embase databases were searched using a combination of subject terms and free words, and the remaining databases were searched using free words.
Participant or population: Patient's disease diagnosed as rare cancer; No limitations on country, ethnicity, gender, economic status and educational background will be implemented.

Intervention: An adaptive clinical trial design was used for the study design.

Comparator: Before adaptive clinical trial design (own pre- and post-control).

Study designs to be included: Including RCT and nonrandomized clinical trials. Studies on animal mechanisms will be excluded. Articles that substantially overlap with another article published in print or electronic media will be excluded. Duplicate publications produced by a single experiment will be published as separate papers based on different criteria for measuring the results, and priority will be given to original publications, while other publications will not be included. There is no limit to the language and time of publication.

Eligibility criteria: Patient's disease diagnosed as rare cancer; The study used an adaptive design, clinical trials with an adaptive design are defined by the FDA as those that allow for prospectively planned modifications to one or more aspects of the design based on accumulating data from subjects in the trial ;The latest progress status of the trial was terminated or completed, and the study type was an interventional study. According to the official Clinicaltrials.gov website, interventional study was defined as a type of clinical study in which participants were assigned to groups that receive one or more intervention/treatment (or no intervention) so that researchers can evaluate the effects of the interventions on biomedical or health-related outcomes.

Information sources: We will manually search five Chinese databases: China National Knowledge Infrastructure (CNKI), China Biology Medicine (CBM), WanFang Data, VIP, and Chinese Clinical Trial Registry (ChiCTR), and five English 
databases: PubMed, The Cochrane Library, Embase, Web of Science, and ClinicalTrials.gov.

Main outcome(s): Primary outcome is the The difference between the actual completion time and the planned completion time of a rare cancer clinical trial.

Quality assessment / Risk of bias analysis: All the included studies will be evaluated based on the guidelines of Cochrane Handbook for Systematic Reviews of Interventions. The quality of each trial will be categorized into 'low', 'unclear', or 'high' risk of bias according to the following items: adequacy of generation of the allocation sequence, allocation concealment, blinding of participants and personal, blinding of outcome assessors, incomplete outcome data, selected reporting the results and other sources of bias (such as comparable baseline).

Strategy of data synthesis: Meta-analysis was performed using RevMan $\mathbf{5 . 3}$ software in Cochrane collaboration network. When outcome indicators are binary data, relative risks (RR) effect quantities were used to express the value. When the outcome index was a continuous variable, the mean difference (MD) or standardized mean difference (SMD) were used to express the outcome index, and the $95 \%$ confidence interval $(\mathrm{Cl})$ and bilateral $Z$ test $P$ value were calculated respectively. $Q$ test and 12 index test were used to test whether there was statistical heterogeneity in the effect quantity. When $12<50 \%$ and $P>0.1$, with not obvious heterogeneity, fixed effect model was used. If $I 2>50 \%$ and $P<0.05$, with a certain degree of heterogeneity, the random effect model could be used in normal distribution. When $12=75 \%-100 \%$, the heterogeneity was obvious. The source of heterogeneity needs to be explored, and subgroup analysis and meta regression were considered. The number of inclusions is greater than or equal to 10 , except in the case of strong clinical heterogeneity, an inverted funnel diagram was drawn to identify publication bias.
Subgroup analysis: We will carry on the subgroup analysis according to the cancer type. If meta-analysis cannot be carried out, we will conduct a descriptive analysis.

Sensitivity analysis: We will conduct a sensitivity analysis to determine the reliability and stability of the summary results by eliminating the tests with high bias risk.

\section{Country(ies) involved: China.}

Keywords: Rare cancer; adaptive design; meta analysis.

Contributions of each author:

Author 1 - Cheng Zhang - Author 1 drafted the manuscript.

Email: zhangc_tcm@foxmail.com

Author 2 - Yue Yang - The author will read, provide feedback and approve the final manuscript.

Email: yanghappy@mail.tsinghua.edu.cn 